\title{
PReS-FINAL-2295: Correlations of health related quality of life reports completed by children with lupus and parents
}

\author{
LN Moorthy ${ }^{1}$, E Roy ${ }^{1}$, MG Peterson², AL Hassett ${ }^{3}$, R Cuttica ${ }^{4}$, C Magalhaes $^{5 *}$, J Sato ${ }^{5}$, S Appenzeller ${ }^{6}$, R Marini ${ }^{7}$, \\ C Len ${ }^{8}$, M Vasco ${ }^{8}$, S Oliveira ${ }^{9}$, M Rodrigues ${ }^{9}$, R Almeida ${ }^{10}$, F Sztajnbok ${ }^{10}$, L Campos $^{11}$, A Jesus ${ }^{11}$, C Silva ${ }^{11}$, \\ E Faugier ${ }^{12}$, L Cobian $^{13}$, A Quintero-Del-Rio ${ }^{13}$, A Adams $^{2}$, L Barinstein $^{14}$, E Chalom $^{15}$, K Onel $^{16}$, J Lopez-Benitez ${ }^{17}$, \\ L Ray ${ }^{18}$, K Haines $^{19}$, P Hashkes ${ }^{20}$, V Cartwright ${ }^{21}$, D Kingsbury ${ }^{21}$, N Singer ${ }^{22}$, I Tomanova-Soltys ${ }^{23}$, S Hong ${ }^{24}$, \\ A Reiff ${ }^{25}$, T Lehman ${ }^{2}$
}

From 20th Pediatric Rheumatology European Society (PReS) Congress

Ljubljana, Slovenia. 25-29 September 2013

\section{Introduction}

Children with chronic diseases and parents often perceive disease impact differently. We previously found moderate correlations between child-parent reports of Simple Measure of Impact of Lupus Erythematosus in Youngsters (SMILEY).

\section{Objectives}

To examine the correlation between child and parent health-related quality of life (HRQOL) scores in an expanded sample from the United States (US) and Latin America (LA).

\section{Methods}

A cross-sectional multicenter cohort of children $(\leq 18$ years) with systemic lupus erythematosus (SLE) and parents completed the specific translation of SMILEY and Pediatric Quality of Life Inventory (PedsQL) Generic scales. Higher scores indicate better HRQOL for both scales. Independent and paired samples t-test were used to compare scores. We examined Spearman's correlation (rho) and intra-class correlation (ICC) between child and parent scores.

\section{Results}

Mean child- (LA $67 \pm 15, \mathrm{n}=123$; US $64 \pm 14, \mathrm{n}=162$ ) and parent-report (LA $64 \pm 16, \mathrm{n}=129$; US $62 \pm 15, \mathrm{n}$ $=148)$ total SMILEY scores were higher for LA subjects.

${ }^{5}$ Pediatrics, Univ. Estadual Paulista (UNESP), Sao Paulo, Brazil

Full list of author information is available at the end of the article
Some SMILEY domain scores ( $p<0.05$ for Effect of Self-child-report; and Social-child- and parent-reports); and child-report PedsQL total scores were also higher for LA subjects. US and LA child-report scores of SMILEY and PedsQL were higher $(\mathrm{p}<0.05)$ compared to corresponding parent-report scores. For child-parent pairs, correlatons ranged from 0.3-0.7 (table 1), with lowest correlation in the Social domain.

\section{Conclusion}

As shown previously, children and parents may have varying attitudes about the impact of SLE on HRQOL. Because parents tend to overestimate disease impact, it remains critical to use both parent and child reports while assessing HRQOL.

\section{Disclosure of interest}

L. Moorthy Grant/Research Support from: Arthritis Foundation Investigator Award supported this study, E. Roy: None declared, M. Peterson: None declared, A.

Table 1 Child-parent correlations from US and LA (p values for rho and ICC $<0$

\begin{tabular}{lll}
\hline Scale and domains & US-rho (n); ICC & LA- rho (n); ICC \\
\hline SMILEY total & $0.5(145) ; 0.5$ & $0.5(122) ; 0.5$ \\
\hline Effect on self & $0.6(144) ; 0.6$ & $0.5(122) ; 0.5$ \\
Limitations & $0.5(145) ; 0.5$ & $0.6(122) ; 0.6$ \\
Social & $0.4(145) ; 0.4$ & $0.3(122) ; 0.3$ \\
Burden of SLE & $0.5(144) ; 0.5$ & $0.4(121) ; 0.5$ \\
\hline Peds QL Generic total & $0.7(138) ; 0.6$ & $0.7(100) ; 0.6$ \\
\hline
\end{tabular}


Hassett Grant/Research Support from: Bristol-Myers Squibb (2013) Pfizer (2012) (not related to current study), R. Cuttica: None declared, C. Magalhaes: None declared, J. Sato: None declared, S. Appenzeller Grant/ Research Support from: Fundacao de Amparo de Pesquisa do Estado de Sao Paulo (FAPESP) 2008/02917-0, Conselho Nacional de Desenvolvimento de Pesquisa CNPQ (471343/2011-0, 302205/2012-8)(not related to current study), R. Marini: None declared, C. Len: None declared, M. Vasco: None declared, S. Oliveira Grant/Research Support from: Roche and Novartis (not related to current study), M. Rodrigues: None declared, R. Almeida: None declared, F. Sztajnbok: None declared, L. Campos: None declared, A. Jesus: None declared, C. Silva: None declared, E. Faugier: None declared, L. Cobian: None declared, A. Quintero-Del-Rio: None declared, A. Adams Grant/ Research Support from: Spoke once for Abbot in 2009 (not related to the current study), L. Barinstein: None declared, E. Chalom: None declared, K. Onel: None declared, J. Lopez-Benitez: None declared, L. Ray: None declared, K. Haines: None declared, P. Hashkes: None declared, V. Cartwright: None declared, D. Kingsbury: None declared, N. Singer: None declared, I. TomanovaSoltys: None declared, S. Hong: None declared, A. Reiff: None declared, T. Lehman: None declared.

\footnotetext{
Authors' details

'Pediatrics, Robert Wood Johnson Medical School-Univ of Medicine and Dentistry of NJ, New Brunswick, NJ, USA. ${ }^{2}$ Hosp. for Special Surgery, New York, NY, USA. ${ }^{3}$ Anesthiology, Univ. of Michigan, Ann Arbor, MI, United States. ${ }^{4}$ Hosp. Pedro de Elizalde, Buenos Aires, Argentina. ${ }^{5}$ Pediatrics, Univ. Estadual Paulista (UNESP), Sao Paulo, Brazil. ${ }^{6}$ Faculty of Medical Science, State Univ. of Campinas, Campinas, Brazil. ${ }^{7}$ Pediatrics, Faculty of Medical Science, State Univ. of Campinas, Campinas, Brazil. ${ }^{8}$ Pediatrics, Universidade Federal de Sao Paulo (UNIFESP)/Escola Paulista de Medicina (EPM), Sao Paulo, Brazil. ${ }^{9}$ Pediatrics, Univ. Federal do Rio de Janeiro, Instituto de Puericultura e Pediatria Martagao Gesteira, University City, Rio de Janeiro, Brazil. ${ }^{10}$ Pediatrics, Univ. do Estado do Rio de Janeiro, Rio de Janeiro, Brazil. ${ }^{11}$ Pediatrics, Children's Institute, Faculdade de Med da Univ. de Sao Paulo, Sao Paulo, Brazil. ${ }^{12}$ Hosp. Infantil de México Federico Gómez, Mexico City, Mexico. ${ }^{13}$ San Jorge Children's Hosp., Santurce, Puerto Rico. ${ }^{14}$ Mount Sinai Hosp, New York City, NY, USA. ${ }^{15}$ St. Barnabas Medical Center, Livingston, NJ, USA. ${ }^{16}$ Univ of Chicago, Chicago, IL, USA. ${ }^{17}$ Centro Medico La Costa, Asuncion, Paraguay. ${ }^{18}$ Univ. of Mississippi Med Center, Jackson, MS, USA. ${ }^{19}$ HUMC, Hackensack, NJ, USA. ${ }^{20}$ Cleveland Clinic Foundation, Cleveland, OH, USA. ${ }^{21}$ Legacy Emanuel Children's Hospital, Portland, OR, USA. ${ }^{22}$ Metro Health Case Western Reserve Univ., Cleveland, OH, USA. ${ }^{23}$ Winthrop University Hosp., Woodmere, NY, USA. ${ }^{24}$ Univ. of lowa, lowa City, IA, USA. ${ }^{25}$ Pediatrics, Children's Hospital Los Angeles, Los Angeles, CA, USA.
}

Published: 5 December 2013

doi:10.1186/1546-0096-11-S2-P285

Cite this article as: Moorthy et al:: PReS-FINAL-2295: Correlations of health related quality of life reports completed by children with lupus and parents. Pediatric Rheumatology 2013 11(Suppl 2):P285.

\section{Submit your next manuscript to BioMed Central and take full advantage of:}

- Convenient online submission

- Thorough peer review

- No space constraints or color figure charges

- Immediate publication on acceptance

- Inclusion in PubMed, CAS, Scopus and Google Scholar

- Research which is freely available for redistribution

Submit your manuscript at www.biomedcentral.com/submit
Ciomed Central 